\title{
Portfolioarbeit im Fremdsprachenunterricht der Grundschule
}

\author{
Annika Kolb (Freiburg/Deutschland)
}

\section{Das Sprachenportfolio in der Grundschule}

In den Fremdsprachenunterricht der Grundschule hat die Portfolioarbeit im Zuge der in Verbindung mit dem Gemeinsamen Europäischen Referenzrahmen für Sprachen entwickelten Idee des Europäischen Sprachenportfolios (vgl. Council of Europe 1997; s. auch Little in diesem Band) Einzug gehalten. In der Folge wurden sowohl in einzelnen Bundesländern als auch im Rahmen des Bund-Länder-Projekts Sprachen lehren und lernen als Kontinuum verschiedene Sprachenportfolios für die Primarstufe entwickelt, die teilweise vom Europarat akkreditiert wurden (vgl. z.B. Kultusministerien der Länder Berlin, Bremen, Hessen, Nordrhein-Westfalen, Thüringen 2008; Ministerium für Bildung, Wissenschaft, Jugend und Kultur RheinlandPfalz 2011 und die Website ${ }^{1}$ des Europarates für eine Übersicht über die akkreditierten Versionen). Auch die meisten Lehrwerke für den Fremdsprachenunterricht der Grundschule enthalten inzwischen Portfolioseiten, auf denen die Kinder aufgefordert werden, ihren Lernfortschritt einzuschätzen und Lernergebnisse zu dokumentieren.

Gerade für die Arbeit in der Grundschule erscheint der Portfolioansatz vielversprechend. Hier wie dort sind individualisiertes Lernen, die Dokumentation subjektiver Lernwege und eine prozessorientierte Sichtweise auf Lernen und Leistung von Bedeutung. So steht in der Primarstufe die pädagogische Funktion der Port-

\footnotetext{
${ }^{1}$ https://www.coe.int/en/web/portfolio/accredited-and-registered-elp [20.02.2019].
} 
folioarbeit im Vordergrund. Ziel ist es, Portfolioarbeit anzubahnen und die Kinder in den Umgang mit diesem Instrument und eine prozessorientierte Lern- und Reflexionskultur einzuführen. Schülerinnen und Schüler erhalten Gelegenheit, die Vielfalt von Sprachen zu erfahren, Sprachen in ihrem Umfeld zu erkunden und werden für Mehrsprachigkeit sensibilisiert. Weiterhin reflektieren sie ihren eigenen Lernprozess und werden angeregt, über das eigene Sprachenlernen, ihre sprachliche Kompetenz, individuelle Zugänge und Lernstrategien nachzudenken und sich darüber auszutauschen. Sprachenportfolios ermöglichen außerdem die Darstellung der eigenen Leistung gegenüber sich selbst und anderen, beispielsweise auch am Übergang von der Primar- in die Sekundarstufe. Diese drei Aspekte - Auseinandersetzung mit Mehrsprachigkeit in der eigenen Lebenswelt, Reflexion des eigenen Lernprozesses und Lernstandes beim Fremdsprachenlernen und Dokumentation der sprachlichen Kompetenzen - spiegeln sich in den verschiedenen Dokumenten, die ein Sprachenportfolio enthalten kann. Gesammelt werden können „Selbsteinschätzungsdokumente [...], individuelle [...] Sprachenbiographien und selbst angefertigte [...] fremdsprachige [...] Arbeitsbeispiele“ (Burwitz-Melzer 2016: 416). Weiterhin werden die drei Dimensionen in den einzelnen Teilen des Sprachenportfolios abgebildet. Auch Portfolioversionen für die Grundschule folgen üblicherweise der vom Europäischen Sprachenportfolio vorgegebenen Gliederung in Sprachenpass, Sprachenbiografie und Dossier. Der Sprachenpass gibt einen Überblick über die Sprachkenntnisse und formalen Qualifikationen seiner Inhaberinnen und Inhaber. In Portfolioversionen für die Primarstufe wird dieser Teil häufig dazu genutzt, Erfahrungen mit Sprachen in der Lebenswelt der Kinder zu dokumentieren. In der Sprachenbiografie schätzen die Lernenden ihre sprachlichen Kompetenzen ein und reflektieren ihren Lernprozess. Es geht um die beiden Fragen $W$ as kann ich? und Wie lerne ich?.2 Im letzten Teil, dem Dossier, werden ausgewählte Schülerarbeiten gesammelt und damit Kompetenzen dokumentiert. Im Folgenden sollen diese drei Dimensionen der Portfolioarbeit im Fremdsprachenunterricht der Grundschule näher beleuchtet werden.

\footnotetext{
2 Beim Vergleich von verschiedenen Portfolioausgaben für die Primarstufe fällt auf, dass die Abgrenzung zwischen Sprachenpass und Sprachenbiografie nicht immer trennscharf ist. Wenn es unter Überschriften wie „Ich und meine Sprachen“ oder „Ich in der Welt“ um Begegnungen mit Sprachen und Kulturen geht, wird das entweder dem Sprachenpass oder der Sprachenbiografie zugeordnet, zuweilen wird ganz auf einen Sprachenpass verzichtet (vgl. z.B. BLK-Grundportfolio).
} 

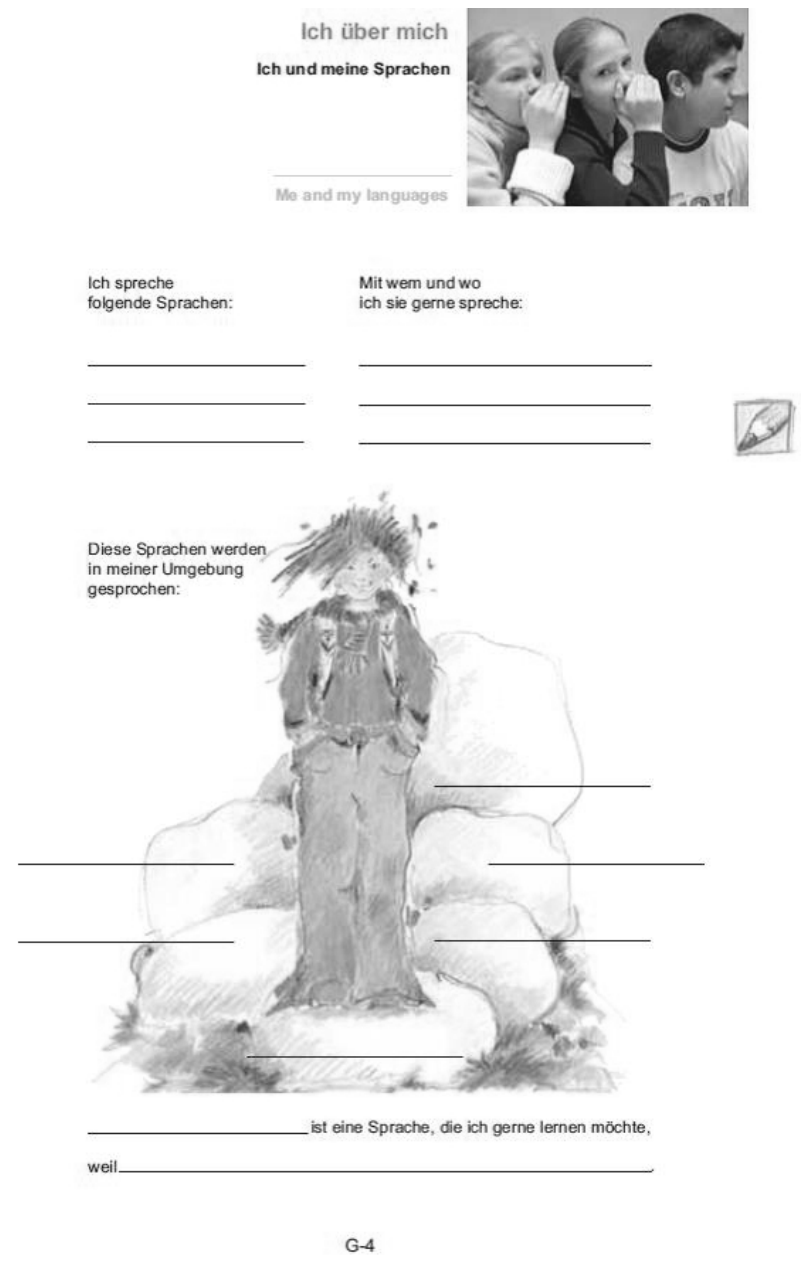

Abb. 1: Bund-Länder-Kommission (BLK)-Portfolio. Grundportfolio, G-4

\section{Mehrsprachigkeit in der eigenen Lebenswelt - Ich und meine Sprachen}

Diese erste Dimension der Portfolioarbeit im Sprachenunterricht der Grundschule zielt auf die Auseinandersetzung mit Mehrsprachigkeit im Umfeld der Kinder (vgl. Abb. 1). Hiermit wird das Ziel des Europäischen Sprachenportfolios verwirklicht, alle Sprachkenntnisse der Inhaber und Inhaberinnen zu dokumentieren, auch solche, die in außerschulischen Kontexten erworben wurden (vgl. Council of Europe 2000). Des Weiteren wird damit im Sinne eines Abschieds vom native speaker model 
auch der Tatsache Rechnung getragen, dass nicht nur fremdsprachliches Können auf einem möglichst hohen Niveau relevant ist (vgl. Rau \& Legutke 2008: 211). Vielmehr werden auch Kompetenzen in einzelnen Fertigkeiten (z.B. nur im rezeptiven Bereich) gewürdigt. Beispielsweise können die Kinder einzelne Wörter, die sie in anderen Sprachen kennen, dokumentieren (vgl. z.B. Ministerium für Bildung, Wissenschaft, Jugend und Kultur Rheinland-Pfalz 2011: 8). Betont wird weiterhin die kommunikative Begegnung mit anderen, was dem Ziel eines intercultural speaker (vgl. Kramsch 1998) entspricht. Sprachliches und kulturelles Lernen werden in einen Zusammenhang gebracht. Es können Eintragungen zu Sprachen, die in der Familie und mit Freunden gesprochen werden, Ländern, in denen Sprachkontakte stattgefunden haben, fremdsprachigen Wörtern und Wendungen, die den Kindern im Alltag begegnen, vorgenommen werden.

Es bietet sich an, zur Einführung in diesen Teil der Portfolioarbeit (oder in die Portfolioarbeit überhaupt) kleine Projekte zum Thema Sprachen und lebensweltliche Mehrsprachigkeit durchzuführen (eine Vielzahl von Anregungen dazu gibt Schader 2012). Durch die Beschäftigung mit der Thematik und ihrer Dokumentation im Portfolio kann der Wert von Mehrsprachigkeit und Plurikulturalität aufgezeigt und die Kinder können zum Lernen weiterer Sprachen motiviert werden, was einer Grundintention des Europäischen Sprachenportfolios entspricht (vgl. Burwitz-Melzer \& Quetz 2006: 203).

\section{Lernprozesse und -ergebnisse reflektieren - Was ich schon kann und wie ich lerne}

Während es im Sprachenpass um eine Vielfalt von Sprachen geht, konzentriert sich die Einschätzung des eigenen Sprachlernprozesses in den Portfolios für die Primarstufe meist auf eine in der Schule gelernte Fremdsprache. Im Zentrum steht die Reflexion des eigenen Lernens, das Nachdenken über das eigene Ich als Lerner(in) (vgl. Kolb 2007: 48). Dieses Nachdenken hat zwei Stoßrichtungen, zum einen die Reflexion der eigenen Kompetenzen (was kann ich?), zum anderen die Reflexion des Sprachlernprozesses (wie lerne ich?).

\subsection{Reflexion der eigenen Kompetenzen}

Die Integration der Lernendenperspektive in die Lernstandserhebung hat zunächst einen diagnostischen Wert. So gibt die Selbsteinschätzung sowohl Schülerinnen und Schülern als auch Lehrkräften eine Rückmeldung über den erreichten Lernstand und kann damit als Basis für das weitere Lernen dienen. Zugleich sollen Lernfortschritte sichtbar gemacht und Sprachenlernen als Prozess erlebt werden (vgl. Becker 2015, Burwitz-Melzer 2008: 171). 
Selbsteinschätzungsfähigkeit stellt außerdem ein Lernziel im Kontext von Sprachlernkompetenz dar. Kinder sollen in die Lage versetzt werden, ihren Lernstand selbständig einzuschätzen, zu dokumentieren, zu diskutieren und zu präsentieren und somit Selbstevaluationskompetenz (vgl. Burwitz-Melzer 2016: 418) zu erwerben. Diese Fähigkeiten sind auch für das Ziel des lebenslangen und des autonomen Sprachenlernens von Bedeutung (vgl. Ballweg 2015: 62-71, Becker 2015, Häcker 2007). Des Weiteren präsentieren Selbsteinschätzungsbögen Lernziele in für die Kinder verständlicher Form. Gerade ein Unterricht, der in den ersten beiden Klassen vorwiegend mündlich durchgeführt wird, hinterlässt bei den Lernenden oft den Eindruck von Flüchtigkeit. Hier kann die Portfolioarbeit bei den Kindern dazu beitragen, sich der eigenen Kompetenzen - vor allem im rezeptiven Bereich - deutlicher bewusst zu werden und Lernergebnisse greifbarer zu machen.

Wie realistisch ist die Einschätzung der Kinder? Das ist eine in diesem Zusammenhang oft gestellte Frage. Selbstverständlich ist von Lernanfängerinnen und Lernanfängern zunächst nicht in jedem Fall ein valides Urteil über ihr Können zu erwarten, auch wenn Selbsteinschätzung von Kindern oft spontan vorgenommen wird und somit jegliche Arbeitsprozesse begleitet. Die Fähigkeit zu einer angemessenen Einschätzung bildet sich vielmehr im Laufe der Zeit heraus. In einem Forschungsprojekt zur Portfolioarbeit (vgl. Kolb 2007: 197-222) zeigte sich, dass die Kinder verschiedene Strategien benutzen, um ihr sprachliches Können einzuschätzen:

- Rückgriff auf das Unterrichtsgeschehen: Die Kinder machen ihr Können am erlebten Unterricht fest und setzen „können“ mit im Unterricht „behandelt haben" gleich.

- Sie suchen nach Fremdbestätigung durch die Lehrkraft oder Mitschüler.

- Sie führen einen Selbsttest durch, in dem sie sich die gefragte Kompetenz selbst unter Beweis stellen.

- Sie mobilisieren ein inneres Bild der eigenen Kompetenz, das durch den Unterricht aufgebaut wurde.

Diese Strategien entsprechen in unterschiedlichem Maße den Zielen der Portfolioarbeit. Nur die beiden Strategien Selbsttest und inneres Bild lenken den Blick auf das individuelle Können und stellen eine Selbsteinschätzung dar, die unabhängig vom Urteil anderer ist und ein differenziertes Bild der eigenen Kompetenz liefert. Lehrkräfte sollten bei der Einführung von Portfolioarbeit demnach darauf gefasst sein, dass die Kinder auch weniger geeignete Strategien zur Einschätzung ihres Sprachkönnens heranziehen und die Selbsteinschätzung zu Beginn deshalb oft wenig verlässlich ist. Die Fähigkeit, eigene Lernergebnisse einzuschätzen, muss also sorgfältig eingeführt und geübt werden.

Um die Entwicklung von Selbsteinschätzungskompetenz zu unterstützen, ist es wichtig, den Kindern das Spezifische der Portfolioarbeit immer wieder zu verdeutlichen und deren Wert für ihr eigenes Lernen herauszustellen. Dazu gehört insbe- 
sondere die Tatsache, dass eine subjektive Einschätzung des eigenen Könnens von ihnen erwartet wird und dass das Portfolio eine Hilfe für ihre persönliche Lernplanung ist. Die Entstehung einer Testsituation sollte vermieden werden. Dazu trägt auch bei, dass Lehrkräfte das Portfolio als Eigentum der Schülerinnen und Schüler respektieren und keine Korrekturen darin vornehmen. Vielmehr sollten sie den Lernenden beim Bearbeiten beratend zur Seite stehen.

Gefördert werden kann die Ausbildung von Selbsteinschätzungsfähigkeit auch durch die Thematisierung von Verfahren und Kriterien der Selbsteinschätzung. Die Kinder können sich darüber austauschen, wie sie zu ihrer Einschätzung gekommen sind, es können gemeinsam Kriterien erarbeitet und Überprüfungsmöglichkeiten des eigenen Könnens (leise vorsprechen, Dialog mit Mitschülerinnen und Mitschülern etc.) ausprobiert werden. Als Einstieg in die Selbsteinschätzung kann am Ende einer Unterrichtsstunde oder einer thematischen Einheit mit einem kurzen Reflexionsgespräch über die Lerninhalte begonnen werden, in dem die Kinder beispielsweise erzählen, was ihnen vom Unterrichtsgeschehen im Gedächtnis geblieben ist, was sie besonders leicht oder schwierig fanden oder was ihnen besonders gefallen hat. Damit wird die Fähigkeit geübt, Lernergebnisse und -prozesse zu reflektieren. Eine weitere Möglichkeit, das Nachdenken über den individuellen Lernprozess anzubahnen, ist es, in regelmäßigen Abständen das eigene Heft zu betrachten und Eintragungen mit bunten Klebepunkten zu markieren (z.B. rot für „,das fand ich schwierig“, blau für „das hat mir besonders gefallen“, orange für „das ist mir besonders gut gelungen“ "etc.).

Zur Ausbildung einer realistischen Selbsteinschätzung gehört weiterhin der Abgleich mit einer Fremdeinschätzung. Diese kann von der Lehrperson oder von Mitschülerinnen und Mitschülern kommen. Die Lehrkraft kann in regelmäßigen Abständen die Portfolios einsammeln und den Lernenden ein Feedback geben. Dabei ist es wichtig, das Urteil der Kinder zunächst ernst zu nehmen und nur vorsichtige Anregungen zu seiner Überprüfung zu geben (z.B. „Bist du dir wirklich sicher, dass du den Reim auswendig kannst? ${ }^{\prime}$. Hier können Lehrende auch Vorschläge für das weitere Lernen machen, wenn sie etwa auf in der Klasse vorhandenes Freiarbeitsmaterial hinweisen.

Empirische Ergebnisse zeigen, dass bei einem solchen Vorgehen eine angemessene Selbsteinschätzung auch schon bei Grundschulkindern ein realistisches Ziel ist (vgl. Kolb 2007, Legutke 2003).

Eine zuverlässige Einschätzung der eigenen Kompetenz hängt gerade bei Lernenden in der Primarstufe von möglichst konkreten, auf die Unterrichtsinhalte zugeschnittenen Deskriptoren ab. Bei der Entwicklung von Portfoliofassungen für die Grundschule ergibt sich hier ein gewisses Dilemma: Auf der einen Seite sollen die Deskriptoren sich auf diejenigen des GER beziehen, um Vergleichbarkeit und eine Einordnung in die entsprechenden Niveaustufen zu gewährleisten. Auf der anderen Seite entsteht dabei ein Abstraktionsgrad, der für Lernende in der Primarstufe die Einschätzung ihrer sprachlichen Kompetenzen nicht mehr ermöglicht (vgl. Burwitz-Melzer 2008: 179f.). Es ist demnach nötig, die Deskriptoren für die 
Kinder durch konkrete sprachliche Handlungen zu veranschaulichen (z.B. „Ich kann sagen, was ich am liebsten esse und trinke“), auch wenn diese eine gewisse Interpretation der ursprünglichen Deskriptoren darstellen.

Um Sprachenlernen als Prozess erlebbar zu machen, ist es sinnvoll, Einträge an mindestens zwei Bearbeitungszeitpunkten zu ermöglichen. Für die Kinder werden der eigene Lernfortschritt oder auch eventuelle Rückschritte sowie die Nachhaltigkeit von Lernen so unmittelbar sichtbar. Die beiden Bearbeitungszeiträume können entweder durch zwei Spalten oder durch unterschiedliche Farben verdeutlicht werden. Dazu gehört auch zu vermitteln, dass nicht alle Inhalte bei der ersten Bearbeitung beherrscht werden müssen. Vielmehr werden die einzelnen Themen im Laufe der Grundschulzeit immer wieder aufgegriffen und die Kompetenzen der Kinder so sukzessive erweitert. Wenn Items vorgegeben werden, sollte immer Platz für die Ergänzungen der Kinder gelassen werden, um persönlich relevante Wörter und Wendungen einzutragen.

\subsection{Reflexion des eigenen Lernens}

Neben die Einschätzung des eigenen Sprachkönnens tritt bei der Reflexion des eigenen Ichs als Lernerin oder Lerner das Nachdenken über den Sprachlernprozess. Hierbei werden den Lernenden Impulse gegeben, sich über eigene Lernzugänge, -hilfen und -strategien Gedanken zu machen sowie emotionale Aspekte des Sprachenlernens zu thematisieren. Ziel ist die Ausbildung von language learning awareness (vgl. Burwitz-Melzer 2008: 184-187). Hierzu bieten viele Portfoliovorlagen auf den Selbsteinschätzungsbögen Gelegenheit für Eintragungen zu „Was mir bei diesem Thema am besten gefallen hat" oder „Was ich noch schwierig finde“. Die weitere Lernplanung wird unter der Überschrift „Was ich zu diesem Thema sonst noch lernen möchte“ oder „Was ich mir vornehme“ eingetragen.

Zur Entwicklung von Sprachlernkompetenz kann die Portfolioarbeit weiterhin dabei helfen, das Repertoire an Lernstrategien zu erweitern. Das Nachdenken über Lernstrategien und individuelle Zugänge zum Lernen ist für Lernanfängerinnen und Lernanfänger eine recht abstrakte Aufgabe, mit Hilfe von kindgemäßen Formen jedoch auch schon in der Grundschule machbar (vgl. de Leeuw 1997, Kolb 2007, Legutke 2003). Ein erster Schritt kann es sein, sich Aktivitäten beim Sprachenlernen zu vergegenwärtigen (z.B. die Arbeit mit Bildkarten, Geschichten hören, Dialoge mit einem Partner oder einer Partnerin etc.). Diese können dann danach beurteilt werden, inwieweit sie zum Lernerfolg beitragen. Angebahnt werden kann die Reflexion des Lernprozesses auch, indem die Schülerinnen und Schüler sich darüber austauschen, was ihnen beim Lernen hilft (z.B. Verknüpfung mit Bildern, Rückgriff auf Mimik und Gestik). Dabei werden sie allmählich für individuelle Lerntypen sensibilisiert und es werden ihnen weitere Strategien an die Hand gegeben. Wichtig ist es dabei, deutlich zu machen, dass es in diesem Bereich kein „richtig“ oder „falsch“, sondern nur individuelle Zugänge zum Lernen gibt. Das im BLK-Projekt entwickelte Portfolio für die Primarstufe gibt beispielsweise 
für die einzelnen Fertigkeiten verschiedene Lernstrategien vor, und die Schülerinnen und Schüler werden aufgefordert einzuschätzen, inwieweit diese sie beim Lernen unterstützen (vgl. Abb. 2). „Der vom Portfolio vorgezeichnete Ablauf, zunächst die Lernenden über Lerntipps nachdenken zu lassen, dann eigenes Lernverhalten abzufragen, neue Lernziele und Lernstrategien planen zu lassen und schließlich die erreichten Lernziele auch zu kontrollieren, ist auch für junge Lernende schon plausibel“" (Burwitz-Melzer 2008: 184).

Wie ich Sprachen lerne

Hörverstehen

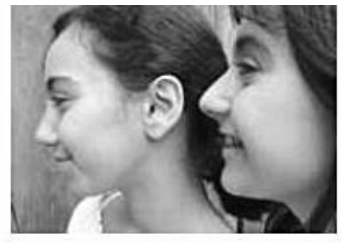

Beim Hörverstehen hilft zum Beispiel,

wenn die Lehrerin oder der Lehrer beim Sprechen Bewegungen macht.

wenn man bekannte Wörter oder Teile von Sätzen wiedererkennt. wenn man etwas gezeigt bekommt (Bilder, Gegenstände).

wenn wichtige Stellen stark betont werden.

\%,

Listening

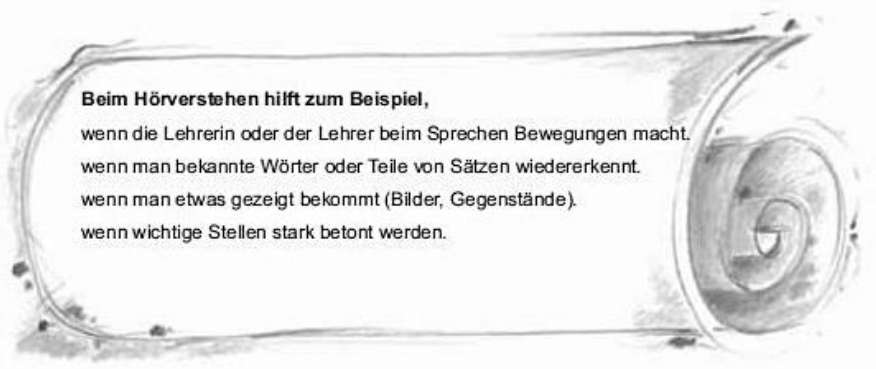

Trage auf der Skala ein, was dir besonders hilft.

Wenn dir etwas sehr hilft, so

Wenn dir etwas wenig hifft, so

\section{Mir hilft,}

wenn ich etwas mehrmals höre.

wenn ich weiß, worum es geht.

wenn

wenn

I

Abb. 2: BLK-Portfolio. Grundportfolio, G-14 


\section{Sprachliche Kompetenzen dokumentieren und präsentieren - Meine Schätze}

Das Dossier - für das in der Grundschule häufig kindgemäßere Begriffe wie „Mein Sprachenschatz“ oder „Schatztruhe“ gewählt werden - sammelt Produkte, die Sprachkönnen dokumentieren (vgl. Rau \& Legutke 2008). Im Sinne eines Produktportfolios steht hier die Präsentation der eigenen Leistung im Vordergrund. Die Kinder sammeln im Dossier „Schätze“, mit denen sie ihr sprachliches Können unter Beweis stellen können (vgl. Dreßler et al. 2016).

Sowohl für Lernende als auch für Lehrende stellt diese Arbeit zunächst eine Herausforderung dar: „Die Lernenden müssen nämlich nicht nur mit dem Konzept des Schatzes vertraut gemacht werden, sondern lernen, Schätze zu entdecken und Schätze zu pflegen“ (Rau \& Legutke 2008: 213). Auswahlkriterien für Dokumente, die in das Dossier aufgenommen werden, müssen im Laufe der Zeit erarbeitet werden. Wichtig ist dabei der Fokus auf das Sprachkönnen: Zu Beginn wählen viele Kinder häufig besonders schön verzierte Arbeitsblätter aus. In diesem Fall sollte den Schülerinnen und Schülern deutlich gemacht werden, dass mit jedem ausgewählten Dokument eine sprachliche Kompetenz unter Beweis gestellt werden sollte. „Der Schatz muss immer aktiver geistiger Besitz des Kindes sein. Er entsteht also aus dem Zusammenwirken von Produkt und sprachlicher Handlung“ (Dreßler et al. 2016: 30). Zur Erarbeitung von Auswahlkriterien sind Kreisgespräche geeignet, in denen Kinder einzelne Dokumente aus ihrem Dossier vorstellen und begründen, warum sie diese ausgesucht haben. Hilfreich ist es außerdem, die Kinder - z.B. auf einem post-it-Zettel - den Satzanfang „Mit dieser Arbeit kann ich zeigen, dass ich ... kann“ vervollständigen zu lassen, z.B. „Mit dieser Seite kann ich zeigen, dass ich Obst und Gemüse auf Englisch benennen kann“ oder „Diese Seite zeigt, dass ich den Reim xy vortragen kann“. Dokumentation und Reflexion stehen hier in einem engen Zusammenhang, da zum einen eine Einschätzung der eigenen Kompetenz und des Lernprozesses notwendig ist, um begründet „Schätze“ auszuwählen. Auf der anderen Seite führen die ausgewählten Dokumente den Lernenden ihre sprachlichen Fähigkeiten deutlich vor Augen, was deren Einschätzung erleichtert.

Neben dem Bezug zur Portfoliodimension Reflexion des eigenen Lernstandes ist auch eine enge Verbindung des Dossiers zur Portfoliodimension Auseinandersetzung mit Mehrsprachigkeit in der eigenen Lebenswelt zu erkennen, da Schätze auch interkulturelle Erfahrungen dokumentieren können, beispielsweise E-Mailprojekte oder Tonaufnahmen eines Interviews in der Erstsprache der Schülerinnen und Schüler.

Ergänzend zur Auswahl von Dokumenten aus dem Unterricht ist es möglich, am Ende einer thematischen Einheit spezielle Arbeiten für das Dossier anzufertigen, die sich dazu eignen, sprachliche Kompetenzen unter Beweis zu stellen. Unter der Überschrift „Was wir zum Thema ... gelernt haben“ können die Schülerinnen und Schüler Bilder zu Geschichten malen und diese dann anhand der Bilder nach- 
erzählen. Kleine Dialoge können - beispielweise in Form von Sprechblasen aufgeschrieben und als Rollenspiel präsentiert werden.

Somit kann das Portfolio, das ja eher ein schriftliches Dokument ist, auch zum Ausgangspunkt für die Präsentation mündlicher Sprachkompetenz werden, die gerade in der Primarstufe im Zentrum des Unterrichts steht. Portfoliodokumente können auf unterschiedliche Weise dazu genutzt werden, Sprechsituationen im Unterricht zu schaffen (vgl. Rau \& Legutke 2008). Die Kinder können auf der Basis ihrer Portfolioeinträge eine kleine Präsentation vorbereiten, beispielsweise anhand eines im Dossier gesammelten fact sheets über das eigene Hobby, und diese der Klasse vorstellen. Dialogisches Sprechen wird eingesetzt, wenn die Kinder sich gegenseitig ihre Schätze vorstellen. Schülerinnen und Schüler müssen immer wieder Gelegenheit bekommen, solche Präsentationen zu üben. Dadurch werden die sprachlichen Mittel gefestigt und ,im Diskurs mit Klassenkameraden und der Lehrperson werden sich die Kinder ihrer Kenntnisse nicht nur bewusster, sondern sie verstärken das Vertrauen in ihr fremdsprachliches Können“" (Rau \& Legutke 2008: 228).

Besonders interessant wird die Inszenierung von Sprechanlässen anhand des Portfolios beim Übergang von der Grundschule in die weiterführende Schule (vgl. Kolb \& Legutke 2016). Den Kindern wird so ermöglicht zu zeigen, was sie können, Lehrkräfte können diese Sprechsituationen zur Diagnose der mündlichen Kompetenz nutzen. Stufenübergreifende Portfolioversionen wie das BLK-Portfolio verdeutlichen den kontinuierlichen Aufbau sprachlicher Kompetenzen. Hier kann das Portfolio eine Brückenfunktion einnehmen.

\section{Herausforderungen bei der Portfolioarbeit}

Trotz des beachtlichen didaktischen Potentials und positiver Erfahrungen im unterrichtlichen Einsatz des Sprachenportfolios der Grundschule (vgl. Kolb 2007, Little et al. 2011) bleibt die flächendeckende Implementierung der Portfolioarbeit im Sprachenunterricht der Primarstufe bis jetzt hinter den Erwartungen zurück (Becker 2013, Little et al. 2011). Als Herausforderungen lassen sich die Einbettung der Portfolioarbeit in den sonstigen Unterricht, ausreichende Unterstützungssysteme für Lehrkräfte und die mit dem Europäischen Sprachenportfolio einhergehende Standardisierung benennen.

Aufschlussreich ist in diesem Zusammenhang eine von Carmen Becker durchgeführte Studie, welche die Implementierung des Sprachenportfolios im Englischunterricht sowohl in Klasse 3 als auch in Klasse 5 in Niedersachsen untersucht (Becker 2013). Sie stellt fest, dass die Mehrzahl der 120 teilnehmenden Lehrkräfte die Portfolioarbeit negativ bewertet und dieses Instrument nicht weiterverwenden möchte. Bei den meisten Lehrerinnen und Lehrern nimmt die Motivation im Verlauf des Projekts ab, die Portfolioarbeit wird eher selten eingesetzt, wenig in den übrigen Unterricht eingebunden und die Möglichkeit der Kooperation mit 
Kolleginnen und Kollegen wird kaum genutzt. Als äußerst relevanten Faktor für eine erfolgreiche Implementierung identifiziert die Autorin eine Anbindung der Portfolioarbeit an den restlichen Unterricht; dem gegenüber geben fast $90 \%$ der Lehrkräfte an, dass sich ihr Unterricht durch den Einsatz von Portfolioarbeit kaum oder gar nicht verändert habe (vgl. Becker 2013: 170). „Eine der Hauptvoraussetzungen einer erfolgreichen und nachhaltigen Portfolioimplementierung ist eine Veränderung der gewohnten Unterrichtsskripte und somit eine Veränderung der Lern- und Gesprächskultur im Vorfeld oder ein Wunsch nach Veränderung allgemein“ (Becker 2013: 218). Die Erprobung des Sprachenportfolios im Englischunterricht in zwei Grundschulklassen (vgl. Kolb 2007) zeigt ähnliche Befunde. Sowohl Lehrkräfte als auch Schülerinnen und Schüler deuten die Portfolioarbeit im Lichte ihrer bisherigen schulischen Sozialisation. Sie gehen von einem für alle Schülerinnen und Schüler mehr oder weniger gleichen Lernstand aus anstatt einen individualisierenden Blick einzunehmen. Der Lernendenperspektive bei der Leistungseinschätzung wird eher wenig Gewicht beigemessen und die Lernergebnisse stehen im Vordergrund (vgl. Kolb 2007: 318-322). Somit wird deutlich, dass Portfolioarbeit ihr Potenzial nicht als isolierte Einzelmaßnahme in einem ansonsten traditionellen Unterricht entfalten kann, sondern nur wenn sie - wie der Titel von Beckers Studie sagt - als ein „Baustein einer neuen Lernkultur“ eingesetzt wird. Diese ist durch Individualisierung, Prozess- statt nur Ergebnisorientierung, Aufwertung der Lernendenperspektive und Kooperation zwischen Lehrkräften gekennzeichnet.

Ebenfalls von entscheidender Bedeutung ist die Unterstützung, die Lehrkräften bei der Einführung der Portfolioarbeit zur Verfügung steht. Sowohl der Bericht des Europarats (vgl. Little et al. 2011) als auch die Studie von Becker sehen diese als unabdingbar für den Erfolg von Portfolioarbeit an. Sie muss über organisatorische Aspekte hinausgehen und etwa Gelegenheit zu individueller Begleitung, Austausch über Erfahrungen oder best practice-Beispiele anbieten (vgl. Becker 2015: 273).

Als problematisch hat sich weiterhin in manchen Fällen die Standardisierung des Europäischen Sprachenportfolios herausgestellt. In vielen Klassen steht die Bewertung des eigenen Lernstands in der in der Schule gelernten Fremdsprache durch die Schülerinnen und Schüler mit Hilfe von Selbsteinschätzungsbögen im Zentrum der Portfolioarbeit. Auch Lehrwerke konzentrieren sich unter der Überschrift „Portfolio“ häufig auf die Selbsteinschätzung des eigenen Könnens. Damit wird das volle Potential der Portfolioarbeit aber bei weitem nicht ausgeschöpft. Die Reflexion von Lernwegen und subjektiven Zugängen zum Sprachenlernen sowie lebensweltliche Mehrsprachigkeit wird hierbei vernachlässigt. Die vorgegebenen Portfoliobögen des Sprachenportfolios scheinen die Ergebnisorientierung zu befördern und Lehrkräften wenig Gelegenheit zur flexiblen Anpassung an die Bedürfnisse ihrer Lerngruppe zu bieten (vgl. Bräuer 2006: 260). So halten $70 \%$ der Lehrkräfte in Beckers Studie die Portfolioarbeit nicht dafür geeignet, Lernprozesse besser zu verstehen, Lernstrategien sichtbar zu machen und individuelle Förder- 
maßnahmen zu entwickeln (vgl. Becker 2013: 219). Es besteht ein gewisses Spannungsfeld zwischen der angestrebten Individualisierung und der Standardisierung bei der Portfolioarbeit.

Die extern vorformulierten Anforderungen in den Selbsteinschätzungsbögen [...] [beinhalten] die Möglichkeit zu Leistungsvergleichen und gehen unter Umständen gerade an den Stärken des individuellen Lernenden vorbei, da sie den eigenen Lernstand mit extern gesetzten Standards in Verbindung bringen (Kolb 2007: 319).

Für eine erfolgreiche Portfolioarbeit ist deshalb gerade die Arbeit mit den „freieren"Teilen - dem Dossier und eigenen Vorlagen - wichtig.

\section{$6 \quad$ Fazit}

Über die drei Dimensionen a) Auseinandersetzung mit Mehrsprachigkeit in der eigenen Lebenswelt, b) Reflexion des eigenen Sprachenlernens und c) Dokumentation und Präsentation sprachlicher Kompetenzen eröffnet die Portfolioarbeit im Sprachenunterricht der Grundschule einen Reflexionsraum und bietet die Chance, language learning awareness zu entwickeln. Gestärkt werden kann weiterhin die Perspektive der Lernenden sowie eine Fokussierung auf das Können anstatt auf die Defizite der Schülerinnen und Schüler. Diese Beschäftigung mit dem individuellen Lernen „konturiert das Selbstbild der Lernenden und stärkt ihr Selbstbewusstsein“ (Rau \& Legutke 2008: 212). Erfolgreich kann Portfolioarbeit vor allem in einem Unterricht sein, der einen individualisierenden Blick auf Schülerinnen und Schüler einnimmt, die Perspektive der Lernenden ernst nimmt, individuelle Lernwege zulässt und den Lernprozess und nicht nur Lernergebnisse würdigt.

\section{Literatur}

Ballweg, Sandra (2015): Porfolioarbeit im Fremdsprachenunterricht. Tübingen: Narr.

Becker, Carmen (2013): Portfolio als Baustein einer neuen Lernkultur. Eine empirische Studie zur Implementierung des Europäischen Portfolios der Sprachen. Frankfurt/M.: Lang.

Becker, Carmen (2015): Assessment and portfolios. In: Bland, Janice (Hrsg.): Teaching English to Young Learners. Critical Issues in Language Teaching with 3-12 Year Olds. London: Bloomsbury, 261-278.

Bräuer, Gerd (2006): Keine verordneten Hochglanz-Portfolios, bitte! Die Korruption einer schönen Idee? In: Brunner, Ilse; Häcker, Thomas; Winter, Felix (Hrsg.): Das Handbuch Porfolioarbeit. Konzepte, Anregungen, Erfahrungen aus Schule und Lehrerbildung. Seelze: Kallmeyer, 257-261. 
Burwitz-Melzer, Eva (2008): Ein neues Portfolio für den Fremdsprachenunterricht: Übergang und Selbstevaluation. In: Grau, Maike; Legutke, Michael K. (Hrsg.): Fremdsprachen in der Grundschule. Auf dem Weg zu einer neuen Lern- und Leistungskultur. Frankfurt: Arbeitskreis Grundschule, 170-193.

Burwitz-Melzer, Eva (2016): Sprachenportfolio. In: Burwitz-Melzer, Eva; Mehlhorn, Grit; Riemer, Claudia; Bausch, Karl-Richard; Krumm, Hans-Jürgen (Hrsg.): Handbuch Fremdsprachenunterricht. 6. vollst. überarb. u. erw. Aufl. Tübingen: Francke, 416-420.

Burwitz-Melzer, Eva; Quetz, Jürgen (2006): Mehrsprachigkeit und Interkulturalität in Fremdsprachenportfolios. In: Martinez, Hélène; Reinfried, Marcus (Hrsg.): Mehrsprachigkeitsdidaktik gestern, heute und morgen. Tübingen: Narr, 203-213.

Council of Europe (1997): European Language Portfolio. Proposals for Development. Strasbourg: Council for Cultural Cooperation.

Council of Europe (2000): The European Language Portfolio. A Guide for Teachers and Teacher Trainers. Strasbourg: Council of Europe.

De Leeuw, Howard (1997): English as a Foreign Language in the German Elementary School: What Do the Children Have to Say? Tübingen: Narr.

Dreßler, Constanze; Kolb, Annika; Kollmann, Sabine; Legutke, Michael K. (Hrsg.) (2016): Herausforderung Übergang: Kontinuität im Englischunterricht. Handreichungen für die Praxis in den Klassen 4 und 5. Braunschweig: Diesterweg.

Dreßler, Constanze; Kollmann, Sabine; Legutke, Michael (2016): Show what you know - show what you can do. Zur Arbeit mit Schätzen. In: Dreßler, Constanze; Kolb, Annika; Kollmann, Sabine; Legutke, Michael K. (Hrsg.): Herausforderung Übergang: Kontinuität im Englischunterricht. Handreichungen für die Praxis in den Klassen 4 und 5. Braunschweig: Diesterweg, 30-41.

Häcker, Thomas (2007): Portfolio: ein Entwicklungsinstrument für selbstbestimmtes Lernen. Hohengehren: Schneider.

Kolb, Annika (2007): Portfolioarbeit. Wie Grundschulkinder ibr Sprachenlernen reflektieren. Tübingen: Narr.

Kolb, Annika; Legutke, Michael (2016): Chancen und Herausforderungen des Englischunterrichts am Übergang von Klasse 4 nach Klasse 5. In: Dreßler, Constanze; Kolb, Annika; Kollmann, Sabine; Legutke, Michael K. (Hrsg.): Herausforderung Übergang: Kontinuität im Englischunterricht. Handreichungen für die Praxis in den Klassen 4 und 5. Braunschweig: Diesterweg, 9-13.

Kramsch, Claire (1998): The privilege of the intercultural speaker. In: Byram, Michael; Fleming, Michael (Hrsg.): Language Learning in Intercultural Perspective: Approaches through Drama and Ethnography. Cambridge: Cambridge University Press, 16-31. 
Kultusministerien der Länder Berlin, Bremen, Hessen, Nordrhein-Westfalen (2008): BLK-Portfolio. Europäisches Portfolio der Sprachen. Grundportfolio. Braunschweig: Diesterweg, Leipzig: Klett, Berlin: Cornelsen.

Legutke, Michael (2003): Neue Wege für die Lernstandsermittlung im fremdsprachlichen Unterricht der Grundschule? Anmerkungen zum Junior-Portfolio für Sprachen. In: Hermes, Liesel; Klippel, Friederike (Hrsg.): Früher oder später: Englisch in der Grundschule und bilingualer Sacbfachunterricht. Berlin: Langenscheidt, 69-86.

Little, David; Goullier, Francis; Hughes, Gareth (2011): The European Language Porffolio. The story so far (1991-2011). Strasbourg: Council of Europe.

Ministerium für Bildung, Wissenschaft, Jugend und Kultur Rheinland-Pfalz (2011): Mein Sprachenportfolio Englisch. Stuttgart: Klett.

Rau, Natalie; Legutke, Michael K. (2008): Sprachliches Können dokumentieren und präsentieren. Anmerkungen zur Arbeit mit dem Dossier (der „Schatztruhe") des Juniorportfolios. In: Grau, Maike; Legutke, Michael K. (Hrsg.): Fremdsprachen in der Grundschule: Auf dem Weg zu einer neuen Lern- und Leistungskultur. Frankfurt: Grundschulverband, 211-229.

Schader, Basil (2012): Sprachenvielfalt als Chance. Zürich: Orell Füssli. 